\title{
CARTAN MATRICES, FINITE GROUPS OF QUATERNIONS, AND KLEINIAN SINGULARITIES ${ }^{1}$
}

\author{
JOHN MCKAY
}

To H.S.M. Coxeter for his 70 th birthday

\begin{abstract}
The eigenvectors of the Cartan matrices of affine type $\bar{A}_{r}, \bar{D}_{r}, \bar{E}_{6}, \bar{E}_{7}$, and $\bar{E}_{8}$ can be taken to be the columns of the character tables of the finite groups of quaternions.
\end{abstract}

Proposition 1. The Cartan matrices of affine type $\bar{A}_{r}, \bar{D}_{r}, \bar{E}_{6}, \bar{E}_{7}$, and $\bar{E}_{8}$ are positive semidefinite. Their eigenvectors can be taken to be the columns of the character table of the corresponding finite group of quaternions [3, Chapters 6, 7], namely the cyclic group $Z_{r+1}$, the binary dihedral (or dicyclic) group of order $4 r-8$, the binary tetrahedral, binary octahedral, and binary icosahedral group respectively.

We remark that any matrix with such eigenvectors necessarily commutes with the matrices of the regular representation of the representation algebra of the appropriate finite group $G$. Only for $\bar{A}_{0}, \bar{A}_{1}$ and $\bar{E}_{8}$ are the eigenvalues simple and each eigenvector determined to within a scalar multiple.

For any (complex) representation $R$ of $G$, we construct a graph $\Gamma_{R}$ with the irreducible representations of $G$ as its nodes and $m_{j k}$ (possibly zero) directed edges from $R_{j}$ to $R_{k}$ where $R \otimes R_{j}=\bigoplus_{k} \dot{m}_{j k} R_{k}$. We convene that an undirected edge between $R_{j}$ and $R_{k}$ represent the pair of directed edges from $R_{j}$ to $R_{k}$ and from $R_{k}$ to $\boldsymbol{R}_{\boldsymbol{j}}$.

Proposition 2. Each of the five types of finite group described above has a faithful two-dimensional representation $R_{Q}$ such that $\Gamma_{R_{Q}}$ is the Coxeter graph of the corresponding affine type.

Taking $R=R_{Q}$ we deduce that the Cartan matrix, $C$, of $\Gamma_{R_{Q}}$ satisfies $C=2 I-$ $M$ where $M=M_{R_{Q}}=\left(m_{j k}\right)$. Proposition 1 follows as does the fact that the eigenvalues of $M$ are the character values afforded by $R_{Q}$.

Singularities on algebraic varieties were studied by Schläfli in 1863 and by Cayley in 1869 although it seems that $\mathrm{Du} \mathrm{Val} \mathrm{[4]} \mathrm{was} \mathrm{the} \mathrm{first} \mathrm{to} \mathrm{relate} \mathrm{the}$ singularities to finite groups. His book [5, \$5.40] contains a description of this relationship in terms of the topology of the spherical neighbourhood of the

Received by the editors June 12, 1979 and, in revised form, November 1, 1979. These results were presented May 24, 1979 at the Coxeter Symposium at the University of Toronto and in expanded form at the American Mathematical Society Finite Groups Conference at Santa Cruz in 1979.

AMS (MOS) subject classifications (1970). Primary 57D45, $20 \mathrm{C15}$.

${ }^{1}$ This research is supported in part by FCAC and NRC research grants. 
singularity. Steinberg [8, p. 156] writes: "Each singularity is realized naturally in the corresponding algebraic group, via a 'ridge' of singularities on the unipotent variety along its subregular subvariety." Brieskorn [1] remarks on the connection with finite groups which is discussed from an algebraic point of view in the forthcoming book of Slodowy [7].

ADDED IN PROOF. The connected undirected graphs with adjacency matrix having maximum eigenvalue 2 are precisely the Coxeter graphs above, together with the graph $A_{\infty}$ which is the representation graph for $S U_{2}$ with $R=R_{Q}$, its natural two-dimensional representation. From this graph one may obtain each of the finite graphs as embeddings by restricting $R$ to a finite subgroup of $S U_{2}$. An interpretation of the dual of the $A_{\infty}$ graph as the Dynkin curve of an appropriate singularity is wanting.

AcKnowledgement. I acknowledge with thanks the help of Dr. David Ford with the computations.

\section{REFERENCES}

1. E. Brieskorn, Singular elements of semi-simple algebraic groups, Actes Congrès Internat. Math., vol. 2, Gauthier-Villars, Paris, 1970, pp. 279-284.

2. A. Cayley, A memoir on cubic surfaces, Philos. Trans. Roy. Soc. London Ser. A 159 (1869), 231-326; Collected works, vol. 6, pp. 359-455.

3. H. S. M. Coxeter, Regular complex polytopes, Cambridge Univ. Press, Cambridge, 1974.

4. P. Du Val, On isolated singularities of surfaces which do not affect the conditions of adjunction, Proc. Cambridge Philos. Soc. 30 (1934), 483-491.

5. __ Homographies, quaternions, and rotations, Clarendon Press, Oxford, 1964.

6. L. Schläfli, On the distribution of surfaces of the third order into species, Philos. Trans. Roy. Soc. London Ser. A 153 (1863), 193-241.

7. P. Slodowy, Simple singularities and simple algebraic groups, Lecture Notes in Math., Springer, New York (to appear).

8. R. Steinberg, Conjugacy classes in algebraic groups, Lecture Notes in Math., vol. 366, SpringerVerlag, Berlin and New York, 1974.

Department of Computer Science, Concordia University, 1455 de Maisonneuve Boulevard West, Montreal, Canada H3G 1 M8 